\title{
Hyperbolic Tangent ansatz method to space time fractional modified KdV, modified EW and Benney-Luke Equations
}

\author{
Ozlem Ersoy Hepson* \\ Eskişehir Osmangazi University, Department of Mathematics \& Computer, 26200, Eskişehir, Turkey.
}

February 16, 2018

\begin{abstract}
The space time fractional Korteweg-de Vries equation, modified Equal Width equation and Benney-Luke Equations are solved by using simple hyperbolic tangent Ansatz method. A simple compatible wave transformation in one dimension is employed to reduce the governing equations to integer ordered ODEs. Then, the ansatz approximation is used to derive exact solutions. Some illustrative examples are presented for some particular choices of parameters and derivative orders.
\end{abstract}

Keywords: Ansatz method; fractional modified Korteweg-de Vries Equation; fractional modified Equal Width Equation; fractional Benney-Luke Equation; Conformable fractional derivative.

MSC2010: 35C07;35R11;35Q53.

PACS: 02.30.Jr; 02.70.Wz; 04.20.Jb

\section{Introduction}

Fractional calculus has been used in almost all fields of engineering, mathematics and other natural sciences. Recently, several mathematical methods have been applied to PDEs. Two-dimensional differential transform method was implemented to obtain approximate analytical solutions of fractional modified Korteweg-de Vries(fmKdV) equation [1]. Homotopy analysis method and its modification were used to solve fmKdV equation [2]. Similarly the homotopy-perturbation method was also capable of getting approximate analytical solution of fmKdV equation [3]. In [4], the travelling wave solutions were expressed in terms of hyperbolic, trigonometric and rational functions. Then $\left(G^{\prime} / G\right)$-expansion method was applied for the analytical solutions of the fmKdV equations. Exact solutions of the fmKdV equation were investigated by the generalized Kudryashov method by Bulut et al. [5]. In that paper, soliton solutions and hyperbolic function solutions were constructed by using the properties of exponential functions.

*ozlemersoy88@gmail.com 
The conformable fractional $\mathrm{mKdV}$ equation

$$
D_{t}^{\alpha} u(x, t)+p u^{2}(x, t) D_{x}^{\beta} u(x, t)+q D_{x}^{3 \beta} u(x, t)=0,
$$

where $u$ is defined for $x>0, t>0$ and the coefficients $p, q \in \mathbb{R}-\{0\}$, is considered. In this equation, the conformal derivative operators $D_{x}^{\beta}$ and $D_{t}^{\alpha}$ represent the $\beta$.th and $\alpha$.th order derivatives $(\beta, \alpha \in(0,1])$.

The fractional modified Equal Width (fmEW) equation in conformable fractional form as

$$
D_{t}^{\alpha} u(x, t)+r u^{2}(x, t) D_{x}^{\beta} u(x, t)+s D_{x t}^{\beta \beta \alpha} u(x, t)=0
$$

is also regarded as the second equation to be solved exactly for $x>0, t>0$. The bright soliton solutions and singular solutions were obtained using ansatz method when the derivatives are in sense of modified Riemann-Lioville derivative [7]. Hosseini and Ayati derived exact solutions of fmEW equation via the Kudryashov method. For this purpose, they introduced fractional complex transformation to reduce it into the integer ordered ordinary differential equation. Korkmaz introduced some more exact solutions in forms of various hyperbolic functions [6].

The space-time fractional Benney-Luke (fBL) equation in conformable fractional form can be interpreted as

$$
\begin{array}{r}
D_{t}^{2 \alpha} u(x, t)-D_{x}^{2 \beta} u(x, t)+\mu D_{x}^{4 \beta} u(x, t) \\
-\eta D_{x t}^{2 \beta 2 \alpha} u(x, t)+D_{t}^{\alpha} u(x, t) D_{x}^{2 \beta} u(x, t) \\
+2 D_{x}^{\beta} u(x, t) D_{x t}^{\beta \alpha} u(x, t)=0
\end{array}
$$

is considered as the last equation to be solved exactly, where $x>0, t>0$ and $\mu$ and $\eta$ are positive parameters, linked to the inverse bond number, whose characteristics is to capture the effects of surface tension and the gravity forces [12]. This equation describes two-way water wave propogation in the presence of surface tension [9]. The existence and analyticicity of lump solutions for generalized Benney-Luke equations was proved by [8]. In [10], some travelling wave solutions of two well-known BL equations were derived via tanh-coth method analytically. An expansion method was applied to the BL equation for travelling wave solutions expressed by the trigonometric, hyperbolic and rational functions in [11]. Also, Kudryashov method and the modified extended tanh expansion were also applied for Benney-Luke equation [13].

This paper is organized as follows: In Section 2, Preliminaries and Essential Tools are given. In section 3, the ansatz method is mentioned briefly. Then, in Sections 4,5,6 the ansatz method is given for space-time fmKdv, fmEW and Benney-Luke equations, respectively. In the last sections,the findings are summerized.

\section{Conformable Fractional Derivative and its Properties}

Conformable fractional derivative of order $\alpha$ th (similarly $\beta$ th) $u$ is described as 


$$
D_{t}^{\alpha}(u(t))=\lim _{\tau \rightarrow 0} \frac{u\left(t+\tau t^{1-\alpha}\right)-u(t)}{\tau}, \alpha \in(0,1]
$$

in the positive half space for a function $u:[0, \infty) \rightarrow \mathbb{R}[14]$. The $\alpha$ th conformable derivative supports several properties given below.

Theorem 1 Let $\alpha \in(0,1]$, and assume that $u$ and $v$ are $\alpha$-differentiable in the positive half plane (interval) $t>0$. Then,

- $D_{t}^{\alpha}(a u+b v)=a D_{t}^{\alpha}(u)+b D_{t}^{\alpha}(v)$

- $D_{t}^{\alpha}\left(t^{p}\right)=p t^{p-\alpha}, \forall p \in \mathbb{R}$

- $D_{t}^{\alpha}(\lambda)=0$, for all constant function $u(t)=\lambda$

- $D_{t}^{\alpha}(u v)=u D_{t}^{\alpha}(v)+v D_{t}^{\alpha}(u)$

- $D_{t}^{\alpha}\left(\frac{u}{v}\right)=\frac{v D_{t}^{\alpha}(u)-u D_{t}^{\alpha}(v)}{v^{2}}$

- $D_{t}^{\alpha}(u)(t)=t^{1-\alpha} \frac{d u}{d t}$

for $\forall a, b \in \mathbb{R}[15-17]$.

The conformable fractional derivative supports many significant properties like Laplace transform, Gronwall's inequality, chain rule, various integration rules, exponential function, and Taylor series expansion [17].

Theorem 2 Let $u$ be an $\alpha$-differentiable function in conformable sense. Also suppose that $v$ is differentiable in classical sense and is defined in the range of $u$. Then,

$$
D_{t}^{\alpha}(u \circ v)(t)=t^{1-\alpha} v^{\prime}(t) u^{\prime}(v(t))
$$

\section{Method of Solution}

The implementation of the ansatz method can be achieved after reducing the fractional PDE

$$
P_{1}\left(u, D_{t}^{\alpha} u, D_{x}^{\beta} u, D_{t t}^{\alpha \alpha} u, D_{x x}^{\beta \beta} u, D_{t x}^{\alpha \beta} u, \ldots\right)=0
$$

to an ODE of the form

$$
P_{2}\left(U, U^{\prime}, U^{\prime \prime}, \ldots\right)=0
$$

by using a compatible wave transformation. In the present study, we choose the wave transformation

$$
u(x, t)=U(\xi), \quad \xi=a\left(\frac{x^{\beta}}{\beta}-\nu \frac{t^{\alpha}}{\alpha}\right)
$$


to satisfy the compatibility of the conformable derivative. The next step is determination of the power $B$ of the ansatz in the predicted solution

$$
U(\xi)=A \tanh ^{B} \xi, A \neq 0, B \neq 0
$$

by substituting it into (7). Setting $B=1$ results in the solution of Eq. (7) leads an algebraic system of equations to specify the relation among the parameters. The particular goal is to determine the parameters $A, a$ and $\nu$ in terms of the others. Once the relations between the parameters are determined, the solution to (7) can be expressed explicitly. The particular goal is to determine the parameters in PDEs in terms of the original variables.

\section{The solutions of the conformable fmKdV Equation}

The wave transformation (8) reduces the fmKdV equation (1)

$$
-a \nu \frac{\mathrm{d}}{\mathrm{d} \xi} U(\xi)+p a U(\xi)^{2} \frac{\mathrm{d}}{\mathrm{d} \xi} U(\xi)+q a^{3} \frac{\mathrm{d}^{3}}{\mathrm{~d} \xi^{3}} U(\xi)=0
$$

Integrating this equation once gives

$$
-a \nu U(\xi)+\frac{1}{3} p a U(\xi)^{3}+q a^{3} \frac{\mathrm{d}^{2}}{\mathrm{~d} \xi^{2}} U(\xi)=K
$$

where $K$ is the constant of integration. Substituting the predicted solution (9) into (11) results in

$$
\begin{aligned}
& -a v A(\tanh (\xi))^{B}+1 / 3 p a A^{3}\left((\tanh (\xi))^{B}\right)^{3}+\frac{q a^{3} A(\tanh (\xi))^{B} B^{2}\left(1-(\tanh (\xi))^{2}\right)^{2}}{(\tanh (\xi))^{2}} \\
& -2 q a^{3} A(\tanh (\xi))^{B} B\left(1-(\tanh (\xi))^{2}\right)-\frac{q a^{3} A(\tanh (\xi))^{B} B\left(1-(\tanh (\xi))^{2}\right)^{2}}{(\tanh (\xi))^{2}}=K
\end{aligned}
$$

If we choose $B=1$, then the previous equation obtained as

$$
\left(\frac{1}{3} p a A^{3}+2 q a^{3} A\right)(\tanh \xi)^{3}+\left(-a v A-2 q a^{3} A\right) \tanh (\xi)=K
$$

Since the predicted solution is different from zero, the last equation is satisfied if only the coefficients of powers of the hyperbolic tangent function are zero. Thus,

$$
\begin{aligned}
\frac{1}{3} p a A^{3}+2 q a^{3} A & =0 \\
-a v A-2 q a^{3} A & =0 \\
K & =0
\end{aligned}
$$


Solution of this algebraic system for $A, a$ and $\nu$ gives

$$
\begin{gathered}
A= \pm \sqrt{-6 \frac{q}{p}} \\
v=-2 q a^{2}
\end{gathered}
$$

for nonzero $p, q$ and arbitrarily chosen $a$. Using these data, one can write the solution of $(7)$ as

$$
u_{1,2}(\xi)= \pm \sqrt{-6 \frac{q}{p}} \tanh (\xi)
$$

Returning the original variables gives the solution to the fmKdV(1) as

$$
u_{1,2}(x, t)= \pm \sqrt{-6 \frac{q}{p}} \tanh \left(a\left(\frac{x^{\beta}}{\beta}+2 \frac{q a^{2} t^{\alpha}}{\alpha}\right)\right)
$$

for nonzero $p, q$. This solution is the general form of hyperbolic tangent function type solution family. Choosing the parameters properly, many solutions in different characteristics covering complex ones can be generated.

Some real solutions are illustrated for particular choices of the parameters. The effect of the derivative order $\alpha$ and $\beta$ to the solution is indicated in the following figures. The solution (15) is chosen to demonstrate a particular solution by choosing the parameters as $p=2$ and $q=-1$. The solutions are plotted on $x t u$-space for various values of $\alpha, \beta$ and $a$. Thus, the solution is reduced to

$$
u(x, t)=-\sqrt{6} \tanh \left(a\left(\frac{x^{\beta}}{\beta}-2 \frac{a^{2} t^{\alpha}}{\alpha}\right)\right)
$$

When the derivative order $\alpha=0.5, \beta=0.5, a=0.5$, the propagation of the wave is in Fig 1(a). When the derivative order $\alpha=0.5, \beta=0.5, a=1$, the propagation of the wave is in Fig 1(b). When the derivative order $\alpha=0.5, \beta=1, a=0.5$, the propagation of the wave is in Fig 2(a). When the derivative order $\alpha=0.5, \beta=1, a=1$, the propagation of the wave is in Fig 2(b). When the derivative order $\alpha=1, \beta=0.5, a=0.5$, the propagation of the wave is in Fig 3(a). When the derivative order $\alpha=1, \beta=0.5, a=1$, the propagation of the wave is in Fig 3(b).

\section{The solutions of the conformable fmEW Equation}

The solution algorithm starts by transforming the fmEW to an ODE by using the compatible wave transformation (8) . Thus, $u(x, t) \rightarrow U(\xi)$. This transformation reduces the fmEW (2) to

$$
-a \nu \frac{\mathrm{d}}{\mathrm{d} \xi} U(\xi)+r a U(\xi)^{2} \frac{\mathrm{d}}{\mathrm{d} \xi} U(\xi)-s a^{3} v \frac{\mathrm{d}^{3}}{\mathrm{~d} \xi^{3}} U(\xi)=0
$$

Integrating both sides of the equation gives 


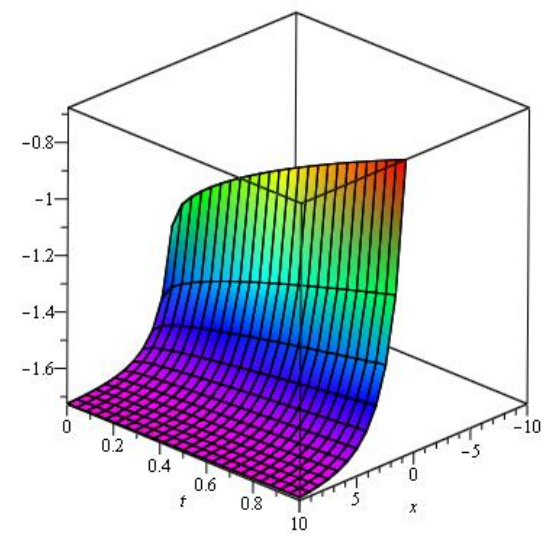

(a)

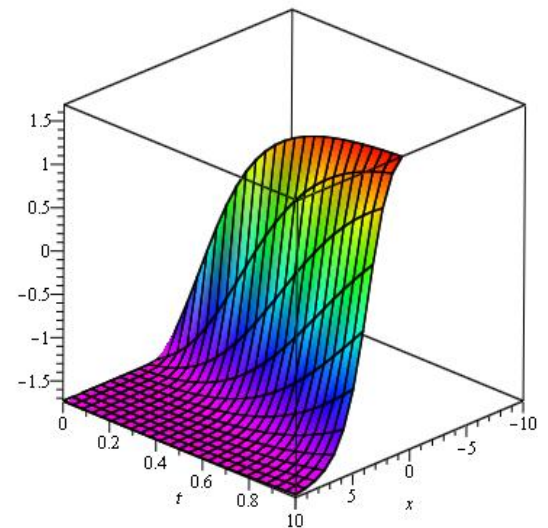

(b)

Figure 1: (a) $\alpha=0.5, \beta=0.5, a=0.5$ (b) $\alpha=0.5, \beta=0.5, a=1$

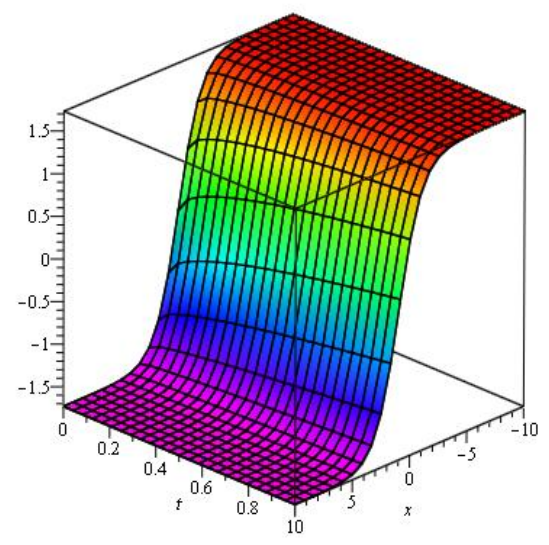

(a)



(b)

Figure 2: (a) $\alpha=0.5, \beta=1, a=0.5$ (b) $\alpha=0.5, \beta=1, a=1$

$$
-a v U(\xi)+\frac{1}{3} r a(U(\xi))^{3}-s a^{3} v \frac{d^{2}}{d \xi^{2}} U(\xi)=K
$$

where $K$ is the constant of integration. Assume that (18) has a solution of the form (9) where $A$ is a nonzero real and $B$ is positive integer. Substituting the predicted solution and its derivatives into the equation (18) gives 




(a)

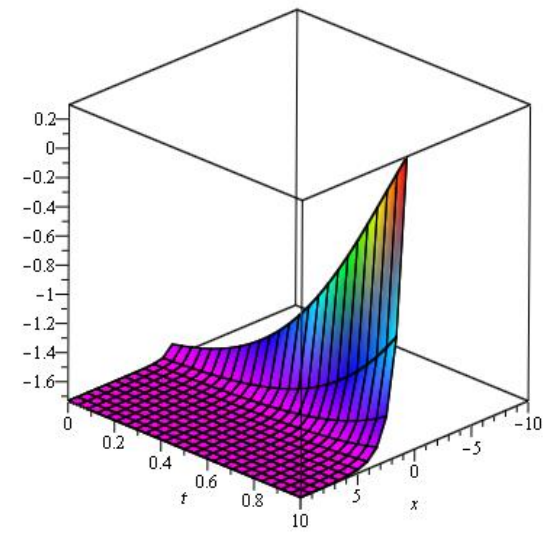

(b)

Figure 3: (a) $\alpha=1, \beta=0.5, a=0.5$ (b) $\alpha=1, \beta=0.5, a=1$

$-a v A(\tanh (\xi))^{B}+\frac{1}{3} r a A^{3}\left((\tanh (\xi))^{B}\right)^{3}-\frac{s a^{3} v A(\tanh (\xi))^{B} B^{2}\left(1-(\tanh (\xi))^{2}\right)^{2}}{(\tanh (\xi))^{2}}$ $+2 s a^{3} v A(\tanh (\xi))^{B} B\left(1-(\tanh (\xi))^{2}\right)+\frac{s a^{3} v A(\tanh (\xi))^{B} B\left(1-(\tanh (\xi))^{2}\right)^{2}}{(\tanh (\xi))^{2}}=K$

$B$ is determined as 1 . Thus, the following equation is obtained as

$$
\left(\frac{1}{3} r a A^{3}-2 s a^{3} v A\right)(\tanh (\xi))^{3}+\left(-a v A+2 s a^{3} v A\right) \tanh (\xi)=K
$$

Collecting the coefficients of powers of the tanh function yields to a system of algebraic equations in $r, s, A, v, a, K$ of the form

$$
\begin{aligned}
\frac{1}{3} r a A^{3}-2 s a^{3} v A & =0 \\
-a v A+2 s a^{3} v A & =0 \\
K & =0
\end{aligned}
$$

is obtained. Solving the resultant system (21-23) for $\{A, a, v, K\}$, the relation among these parameters are stated as

$$
\begin{aligned}
& a= \pm \frac{1}{\sqrt{2 s}} \\
& v=\frac{1}{3} r A^{2}
\end{aligned}
$$


for arbitrarily chosen $r, s$. Thus, the solution to the equation (7) is determined as

$$
u_{3,4}(\xi)=A \tanh (\xi)
$$

Returning the original variables $(x, t)$ from $\xi$ gives the solution to the $\mathrm{EW}(2)$ as

$$
u_{3,4}(x, t)= \pm A \tanh \left(\frac{1}{\sqrt{2 s}}\left(\frac{x^{\beta}}{\beta}-\frac{r A^{2} t^{\alpha}}{3 \alpha}\right)\right)
$$

The plots of the solutions for some fixed values of the parameters $(A=5, r=1, s=1)$ When the derivative order $\alpha=1, \beta=0.2$, the propagation of the wave is in Fig 4(a). When the derivative order $\alpha=1, \beta=1$, the propagation of the wave is in Fig 4(b). When the derivative order $\alpha=0.2, \beta=1$, the propagation of the wave is in Fig 5 (a). When the derivative order $\alpha=0.8, \beta=1$, the propagation of the wave is in Fig $5(\mathrm{~b})$.

$$
U(x, t)=\tanh \left(\frac{1}{2} \sqrt{2} \sqrt{\frac{1}{s}}\left(x-\frac{25}{3} r t\right)\right)
$$

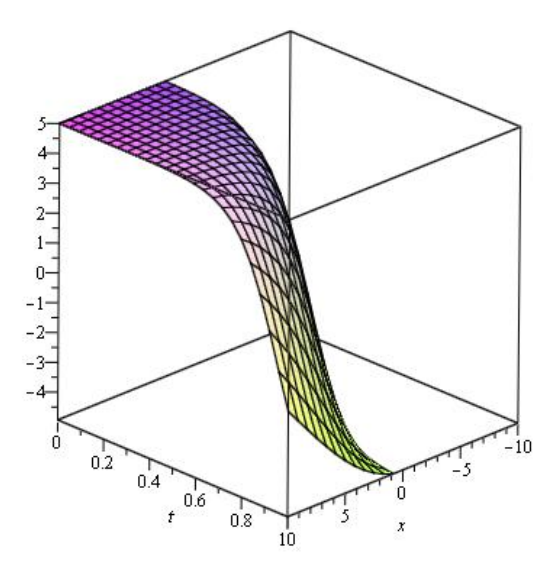

(a)

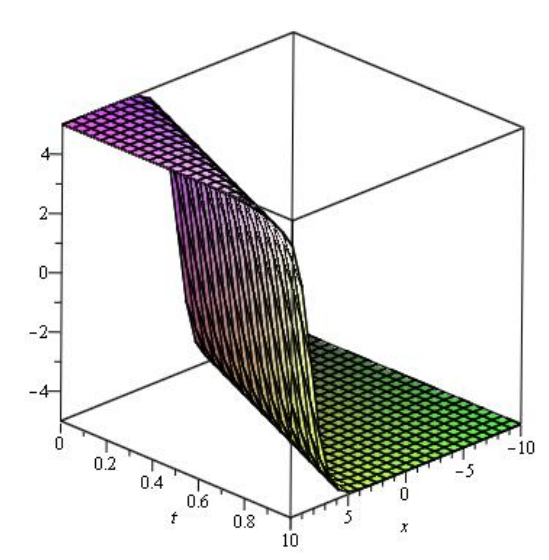

(b)

Figure 4: (a) $\alpha=1, \beta=0.2$ (b) $\alpha=1, \beta=1$

\section{The solutions of the conformable fBL Equation}

The wave transformation (8) reduces the fBL equation (3)

$$
a^{2}\left(\nu^{2}-1\right) \frac{\mathrm{d}^{2}}{\mathrm{~d} \xi^{2}} U(\xi)+a^{4}\left(\mu-\eta v^{2}\right) \frac{\mathrm{d}^{4}}{\mathrm{~d} \xi^{4}} U(\xi)-3 a^{3} v \frac{\mathrm{d}}{\mathrm{d} \xi} U(\xi) \frac{\mathrm{d}^{2}}{\mathrm{~d} \xi^{2}} U(\xi)=0
$$

Integrating this equation once gives

$$
a^{2}\left(\nu^{2}-1\right) \frac{\mathrm{d}}{\mathrm{d} \xi} U(\xi)+a^{4}\left(\mu-\eta v^{2}\right) \frac{\mathrm{d}^{3}}{\mathrm{~d} \xi^{3}} U(\xi)-\frac{3}{2} a^{3} v\left(\frac{\mathrm{d}}{\mathrm{d} \xi} U(\xi)\right)^{2}=K
$$




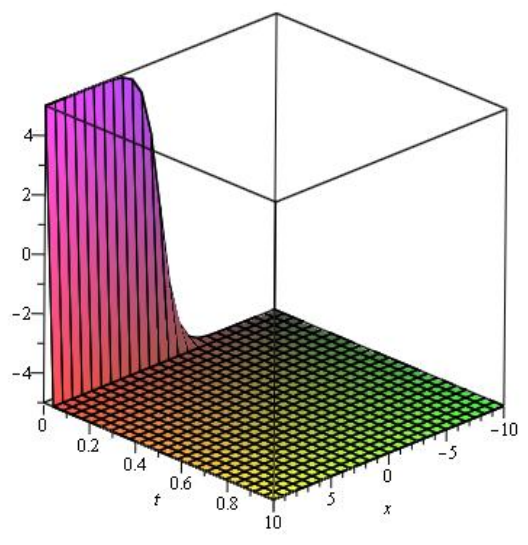

(a)

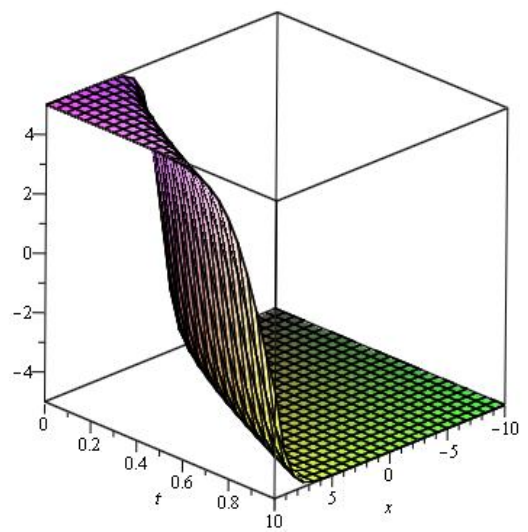

(b)

Figure 5: (a) $\alpha=0.2, \beta=1$ (b) $\alpha=0.8, \beta=1$

where $K$ is the constant of integration. Substituting the predicted solution (9) into (3) results in

$$
\begin{aligned}
& \frac{a^{2} A \tanh (\xi)^{B} B v^{2}}{\tanh (\xi)}-a^{2} v \tanh (\xi)^{B} B \tanh (\xi) v^{2}-\frac{a^{2} A \tanh (\xi)^{B} B}{\tanh (\xi)} \\
+ & a^{2} A \tanh (\xi)^{B} B \tanh (\xi)+\frac{a^{4} \mu A \tanh (\xi)^{B} B^{3}\left(1-\tanh (\xi)^{2}\right)^{3}}{\tanh (\xi)^{3}} \\
& -\frac{6 a^{4} \mu A \tanh (\xi)^{B} B^{2}\left(1-\tanh (\xi)^{2}\right)^{2}}{\tanh (\xi)}-\frac{3 a^{4} \mu A \tanh (\xi)^{B} B^{2}\left(1-\tanh (\xi)^{2}\right)^{3}}{\tanh (\xi)^{3}} \\
& +4 a^{4} \mu A \tanh (\xi)^{B} B \tanh (\xi)\left(1-\tanh (\xi)^{2}\right)+\frac{4 a^{4} \mu A \tanh (\xi)^{B} B\left(1-\tanh (\xi)^{2}\right)^{2}}{\tanh (\xi)} \\
& +\frac{2 a^{4} \mu A \tanh (\xi)^{B} B\left(1-\tanh (\xi)^{2}\right)^{3}}{\tanh (\xi)^{3}}-\frac{a^{4} \eta v^{2} A \tanh (\xi)^{B} B^{3}\left(1-\tanh (\xi)^{2}\right)^{3}}{\tanh (\xi)} \\
& +\frac{6 a^{4} \eta v^{2} A \tanh (\xi)^{B} B^{2}\left(1-\tanh (\xi)^{2}\right)^{2}}{\tanh (\xi)}+\frac{3 a^{4} \eta v^{2} A \tanh (\xi)^{B} B^{2}\left(1-\tanh (\xi)^{2}\right)^{3}}{\tanh (\xi)^{3}} \\
& -4 a^{4} \eta v^{2} A \tanh (\xi)^{B} B \tanh (\xi)\left(1-\tanh (\xi)^{2}\right) \\
& -\frac{4 a^{4} \eta v^{2} A \tanh (\xi)^{B} B\left(1-\tanh (\xi)^{2}\right)}{\tanh (\xi)}-\frac{2 a^{4} \eta v^{2} A \tanh (\xi)^{B} B\left(1-\tanh (\xi)^{2}\right)^{3}}{\tanh (\xi)^{3}} \\
& -\frac{3}{2} \frac{a^{3} v A^{2}\left(\tanh (\xi)^{B}\right)^{2} B^{2}\left(1-\tanh (\xi)^{2}\right)^{2}}{\tanh (\xi)^{2}} \\
= & K
\end{aligned}
$$

$B$ is determined as 1 by. Thus the following equation is get 


$$
\begin{aligned}
& \left(-6 a^{4} \mu A+6 a^{4} \eta v^{2} A-\frac{3}{2} a^{3} v A^{2}\right) \tanh (\xi)^{4} \\
+ & \left(a^{2} A-8 a^{4} \eta v^{2} A+8 a^{4} \mu A-a^{2} A v^{2}+3 a^{3} v A^{2}\right) \tanh (\xi)^{2} \\
& +a^{2} A v^{2}-\frac{3}{2} a^{3} v A^{2}-a^{2} A-2 a^{4} \mu A+2 a^{4} \eta v^{2} A \\
= & K
\end{aligned}
$$

Since the predicted solution is different from zero, the last equation is satisfied if only the coefficients of powers of the hyperbolic tangent function are zero. Thus,

$$
\begin{aligned}
\left(-6 a^{4} \mu A+6 a^{4} \eta v^{2} A-\frac{3}{2} a^{3} v A^{2}\right) & =0 \\
\left(a^{2} A-8 a^{4} \eta v^{2} A+8 a^{4} \mu A-a^{2} A v^{2}+3 a^{3} v A^{2}\right) & =0 \\
a^{2} A v^{2}-\frac{3}{2} a^{3} v A^{2}-a^{2} A-2 a^{4} \mu A+2 a^{4} \eta v^{2} A-K & =0
\end{aligned}
$$

Solution of this algebraic system for $A, a$ and $\nu$ gives

$$
\begin{aligned}
A & = \pm \frac{4(-\mu+\eta) a}{\left(4 a^{2} \eta-1\right) \sqrt{-\frac{1-4 a^{2} \mu}{4 a^{2} \eta-1}}} \\
v & = \pm \sqrt{-\frac{1-4 a^{2} \mu}{4 a^{2} \eta-1}}
\end{aligned}
$$

for nonzero $\mu, \eta$ and arbitrarily chosen $a$. Using these data, one can write the solution of $(7)$ as

$$
u_{5,6,7,8}(\xi)= \pm \frac{4(-\mu+\eta) a}{\left(4 a^{2} \eta-1\right) \sqrt{-\frac{1-4 a^{2} \mu}{4 a^{2} \eta-1}}} \tanh (\xi)
$$

Returning the original variables gives the solution to the space-time fractional BL equation (3)) as

$$
u_{5,6,7,8}(x, t)= \pm \frac{4(-\mu+\eta) a \tanh \left(a\left(\frac{x^{\beta}}{\beta} \pm \sqrt{-\frac{1-4 a^{2} \mu}{4 a^{2} \eta-1}} \frac{t^{\alpha}}{\alpha}\right)\right)}{\left(4 a^{2} \eta-1\right) \sqrt{-\frac{1-4 a^{2} \mu}{4 a^{2} \eta-1}}}
$$

This solution is the general form of hyperbolic tangent function type solution family. Choosing the parameters properly, many solutions in different characteristics covering complex ones can be generated.

The plots of the solutions to $x t$ plane for some fixed values of the parameters $(\mu=3, \eta=$ 2) When the derivative order $\alpha=0.2, \beta=1, a=1$, the propagation of the wave is in Fig 6 (a). When the derivative order $\alpha=0.8, \beta=1, a=1$, the propagation of the wave 
is in Fig $6(\mathrm{~b})$. When the derivative order $\alpha=1, \beta=0.2, a=5$, the propagation of the wave is in Fig 7 (a). When the derivative order $\alpha=1, \beta=1, a=5$, the propagation of the wave is in Fig 7(b).

$$
u(x, t)=-\frac{4(-\mu+\eta) \tanh \left(x-\sqrt{-\frac{1-4 \mu}{4 \eta-1}} t\right)}{(4 \eta-1) \sqrt{-\frac{1-4 \mu}{4 \eta-1}}}
$$

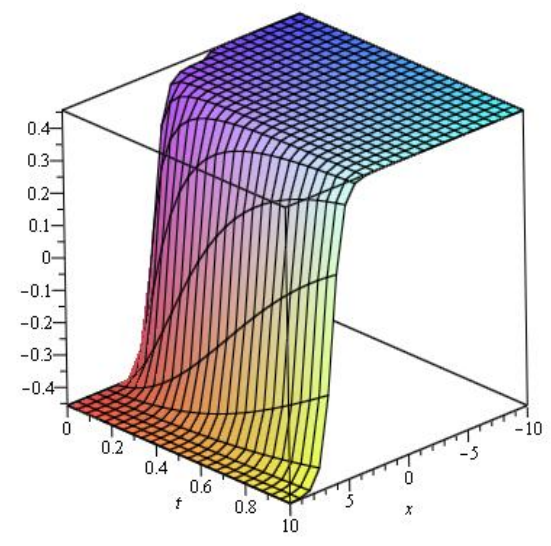

(a)

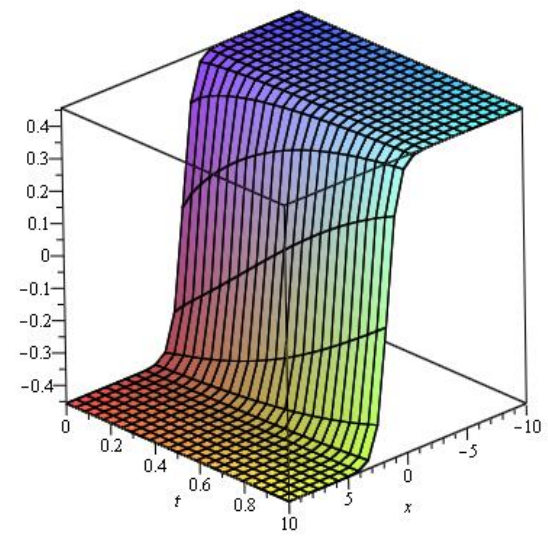

(b)

Figure 6: (a) $\alpha=0.2, \beta=1, a=1$ (b) $\alpha=0.8, \beta=1, a=1$

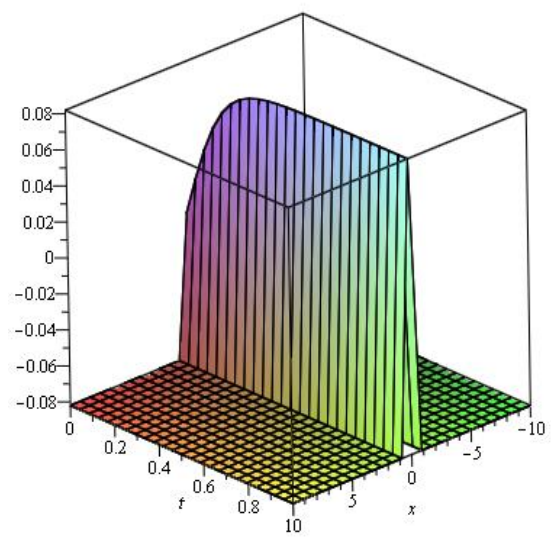

(a)

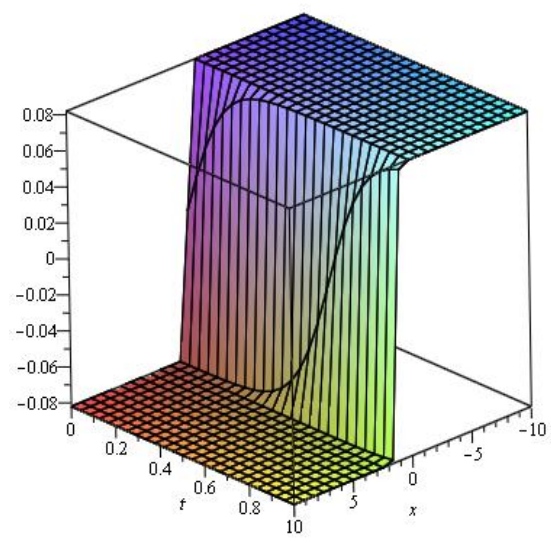

(b)

Figure 7: (a) $\alpha=1, \beta=0.2, a=5$ (b) $\alpha=1, \beta=1, a=5$ 


\section{Conclusion}

Some conformable space-time fractional PDEs are solved by taking hyperbolic tangent ansatz. FmKdV, fmEW and fBL equations are reduced to some nonlinear ODEs of integer order by using compatible wave transformations. Substituting the solution into the resultant ODEs and finding relations between the parameters of the equations give the exact solutions of PDEs. The exact solutions are successfully found for particular choices of the $\alpha$ and $\beta$ and the other parameters.As a conclusion, the method is applicable to PDEs in the theory of fractional calculus.

\section{References}

[1] Kuralay, M., Bayram, M. (2010). Approximate analytical solution for the fractional modified KdV by differential transform method. Commun Nonlinear Sci Numer Simulat 15, 1777-1782.

[2] Abdulaziz, O., Bataineh, A. S., Hashim, I. (2009) On convergence of homotopy analysis method and its modification for fractional modified KdV. J Appl Math Comput 33, 61-81. equations

[3] Abdulaziz, O., Hashim, I., Ismail, E.S. (2009). Approximate analytical solution to fractional modified KdV equations. Mathematical and Computer Modelling 49, $136-145$.

[4] Wang, M., Li, X., Zhang, J. (2008). The (G/G )-expansion method and travelling wave solutions of nonlinear evolution equations in mathematical physics. Physics Letters A 372, 417-423.

[5] Bulut, H., Pandir, Y., Demiray, S. T. (2014). Exact Solutions of Time-Fractional KdV Equations by Using Generalized Kudryashov Method. International Journal of Modeling and Optimization, 4(4), 315-320.

[6] Korkmaz, A. (2017). Exact solutions of space-time fractional EW and modified EW equations. Chaos, Solitons and Fractals 96, 132-138.

[7] Hosseini, K., Ayati, Z. (2016). Exact solutions of space-time fractional EW and modified EW equations using Kudryashov method. Nonlinear Sci. Lett. A, 7(2), $58-66$.

[8] Quintero, J. R. (2002). Existence and analyticity of lump solutions for generalized Benney-Luke equations. Revista Colombiana de Matematicas, 36, 71-95.

[9] Quintero, J. R., Grajales, J. C. M. (2008). Instability of solitary waves for a generalized Benney-Luke equation. Nonlinear Analysis, 68, 3009-3033. 
[10] Gozukızıl, O. F. and Akcağıl, S. (2012). Travelling Wave Solutions to the BenneyLuke and the Higher-Order Improved Boussinesq Equations of Sobolev Type. Abstract and Applied Analysis. 890574, 1-10.

[11] Islam, R. S. M. (2015). Applications of the $\exp (\phi)$-Expansion Method to Find Exact Traveling Wave Solutions of the Benney-Luke Equation in Mathematical Physics. American Journal of Applied Mathematics, 3(3), 100-105.

[12] Khan. U., Ellahi, R. Khan, R., Mohyud-Din S. T. (2017). Extracting new solitary wave solutions of Benny-Luke equation and $\phi-4$ equation of fractional order by using (G/G)-expansion method. Opt Quant Electron (2017) 49, 362.

[13] Ali, K. K., Nuruddeen, R.I. (2017). Analytical treatment for the conformable spacetime fractional Benney-Luke equation via two reliable methods, International Journal of Physical Research, 5(2), 109-114.

[14] Khalil, R., Al Horani, M., Yousef, A., \& Sababheh, M. (2014). A new definition of fractional derivative. Journal of Computational and Applied Mathematics, 264, $65-70$.

[15] Atangana, A., Baleanu, D., \& Alsaedi, A. (2015). New properties of conformable derivative. Open Mathematics, 13(1), 1-10.

[16] Çenesiz, Y., Baleanu, D., Kurt, A., \& Tasbozan, O. (2016). New exact solutions of Burgers' type equations with conformable derivative. Waves in Random and Complex Media, 1-14.

[17] Abdeljawad, T. (2015). On conformable fractional calculus. Journal of computational and Applied Mathematics, 279, 57-66. 